\section{Analysis and Design of Integral Sliding Manifolds for Systems With Unmatched Perturbations}

\author{
Fernando Castaños and Leonid Fridman
}

\begin{abstract}
The robustness properties of integral sliding-mode controllers are studied. This note shows how to select the projection matrix in such a way that the euclidean norm of the resulting perturbation is minimal. It is also shown that when the minimum is attained, the resulting perturbation is not amplified. This selection is particularly useful if integral sliding-mode control is to be combined with other methods to further robustify against unmatched perturbations. $\mathcal{H}_{\infty}$ is taken as a special case. Simulations support the general analysis and show the effectiveness of this particular combination.
\end{abstract}

Index Terms $-\mathcal{H}_{\infty}$, robust control, sliding-mode control (SMC), variable structure systems.

\section{INTRODUCTION}

Sliding-mode control (SMC) [1] is a robust technique, well known for its ability to withstand external disturbances and model uncertainties satisfying the matching condition, that is, perturbations that enter the state equation at the same point as the control input (e.g., the case of completely actuated systems). SMC has other advantages as well, like ease of implementation and reduction in the order of the state equation. The latter property clearly simplifies the control design problem.

Roughly speaking, the conventional SMC design methodology comprises two steps: First, design a sliding manifold such that the system's motion along the manifold meets the specified performance; second, design a (discontinuous) control law, such that the system's state is driven toward the manifold and stays there for all future time, regardless of disturbances or uncertainties. The resulting controller, although robust against matched perturbations, has some disadvantages. Among them, we have: The need to measure the whole state; the lack of robustness against unmatched perturbations; and the reaching phase, i.e., an initial period of time in which the system has not yet reached the sliding manifold and it is sensitive, even to perturbations satisfying the matching condition.

Several strategies have been proposed to solve these problems. See, for example, [2]-[6], where the need to measure the whole state is relaxed. To address the issue of robustness against unmatched perturbations the main strategy has been the combination of SMC with other robust techniques, e.g., [7]-[9].

In order to solve the reaching phase problem an integral slidingmode design concept was proposed [10], [11]. The basic idea is to define the control law as the sum of a continuous nominal control and a discontinuous control. The nominal control is responsible for the performance of the nominal system, i.e., without perturbations; and the discontinuous control is used to reject the perturbations. An integral term is included in the sliding manifold, this guarantees that the system trajectories will start in the manifold from the first time instant.

Manuscript received April 5, 2005; revised August 3, 2005 and December 23, 2005. Recommended by Associate Editor L. Magni. This work was supported by Consejo Nacional de Ciencia y Tecnología (CONACyT) under Grant 43807-Y, and by Programa de Apoyo a Proyectos de Investigación e Innovación Tecnológica (PAPIIT), UNAM, under Grant 117103.

The authors are with the Department of Control, Engineering Faculty, Universidad Nacional Autónoma de México (UNAM), C.P. 04510 Mexico City, Mexico (e-mail: lfridman@verona.fi-p.unam.mx).

Digital Object Identifier 10.1109/TAC.2006.875008

\section{A. Motivation}

To solve the problems of the reaching phase and of the robustness against unmatched perturbations simultaneously (e.g., in the case of subactuated systems), the main idea -as in the conventional slidingmode case- has been the combination of integral sliding-mode control and other robust techniques. The particular combination depends of course on the specific nature of each problem, and each particular combination has a set of details that needs to be properly addressed. In the case of multimodel uncertain systems [12], [13] a multimodel decomposition becomes the essential problem; in the case of nonlinear systems with unknown unmatched uncertainties [14] Lyapunov's direct method becomes a key feature; if integral sliding-mode control is to be combined with LMI-based control techniques, the selection of the equivalent matched dynamics would be the main issue. For systems with time delay the essential problem is that the nominal control should contain a delayed component [15].

In all of the aforementioned cases, the selection of the projection matrix plays a key role in the design of the sliding manifold. In this note we address the need for a universal choice of such matrix. The results are then complemented with an $\mathcal{H}_{\infty}$ approach.

\section{B. Main Contribution}

In this work, we show the following.

- At an integral sliding mode, the discontinuous control completely compensates the matched perturbations, but the unmatched ones are replaced by another (which we will call equivalent) disturbance.

- There is a set of projection matrices for which the norm of the equivalent disturbance is minimal.

- For any projection matrix in this set, the gain of the discontinuous action is also minimal and the equivalent disturbance equals the unmatched one, i.e., there is no amplification of the unmatched disturbance.

All of this means that an integral sliding-mode controller, if improperly designed, while eliminating the matched perturbations, could lead to amplification of the unmatched ones.

The main results are general and can be applied whenever ISMC is to be combined with other techniques to robustify against unmatched disturbances. In this note $\mathcal{H}_{\infty}$ control is taken as a specific case. Simulations support the validity of the analysis developed and show that the performance of an $\mathcal{H}_{\infty}$ controller can be increased by this particular combination.

\section{Paper's Structure}

In the next section, we present a short review of ISMC and state the problem formally. In Section III the problem statement is solved and different interpretations are given to the results. In Section IV we analyze the combination of ISM with $\mathcal{H}_{\infty}$ control. The conclusions are given in Section V.

\section{PROBlem Statement}

\section{A. Preliminaries, ISMC}

Consider a nonlinear system of the form

$$
\dot{x}=f(x, t)+B u(x, t)+\phi(x, t)
$$

where $x \in \mathbb{R}^{n}$ is the state, $t \in \mathbb{R}$ represents time, $u(x, t) \in \mathbb{R}^{m}$ is the control action and $\phi(x, t)$ is a perturbation due to model uncertainties or external disturbances. The following assumptions are made. 
Assumption 1: $\operatorname{rank} B=m$.

Assumption 2: The actual value of $\phi(x, t)$ is of course unknown, but it is bounded by a known function $\bar{\phi}(x, t) \in \mathcal{L}_{\infty}$, i.e., $\|\phi(x, t)\| \leq$ $\bar{\phi}(x, t)$ for all $x$ and $t$.

In the ISMC approach, a law of the form

$$
u(x, t)=u_{0}(x, t)+u_{1}(x, t)
$$

is proposed. The nominal control $u_{0}(x, t)$ is responsible for the performance of the nominal system; $u_{1}(x, t)$ is a discontinuous control action that rejects the perturbations by ensuring the sliding motion. The sliding manifold is defined by the set $\{x \mid s(x, t)=0\}$, with

$$
s(x, t)=G\left[x(t)-x\left(t_{0}\right)-\int_{t_{0}}^{t}\left(f(x, \tau)+B u_{0}(x, \tau)\right) \mathrm{d} \tau\right] .
$$

$G \in \mathbb{R}^{m \times n}$ is a projection matrix which must satisfy Assumption 3: The matrix product $G B$ is invertible.

The term

$$
x\left(t_{0}\right)+\int_{t_{0}}^{t}\left(f(x, \tau)+B u_{0}(x, \tau)\right) \mathrm{d} \tau
$$

in (2) can be thought as a trajectory of the system in the absence of perturbations and in the presence of the nominal control $u_{0}$, that is, as a nominal trajectory for a given initial condition $x\left(t_{0}\right)$. With this remark in mind, $s(x, t)$ can be considered a penalizing factor of the difference between the actual and the nominal trajectories, projected along $G$ (hence, the name projection matrix, not to be confused with a projection operator). Notice that at $t=t_{0}, s(x, t)=0$, so the system always starts at the sliding manifold.

The discontinuous control $u_{1}$ is usually selected as

$$
u_{1}(x, t)=-\rho(x, t) \frac{(G B)^{T} s(x, t)}{\left\|(G B)^{T} s(x, t)\right\|}
$$

where $\rho(x, t)$ is a gain high enough to enforce the sliding motion. To simplify notation, we will omit some of the functions' arguments from now on.

\section{B. Analysis of the Unmatched Perturbation}

Before we analyze the effect of the unmatched perturbation, it is convenient to introduce the following proposition.

Proposition 1: For any matrix $B \in \mathbb{R}^{n \times m}$ satisfying Assumption 1 , the identity

$$
I_{n}=B B^{+}+B^{\perp} B^{\perp+}
$$

holds, where $B^{+}$is understood as the left inverse of $B$, that is $B^{+}=$ $\left(B^{T} B\right)^{-1} B^{T}$ and the columns of $B^{\perp} \in \mathbb{R}^{n \times(n-m)}$ span the null space of $B^{T}$.

Proof: Consider a matrix

$$
P=\left[\begin{array}{c}
B^{+} \\
B^{\perp+}
\end{array}\right]
$$

This matrix is clearly nonsingular since it's inverse is given by $P^{-1}=$ $\left[\begin{array}{ll}B & B^{\perp}\end{array}\right]$, that is

$$
P \cdot P^{-1}=\left[\begin{array}{cc}
B^{+} B & 0 \\
0 & B^{\perp+} B^{\perp}
\end{array}\right]=\left[\begin{array}{cc}
I_{\mathrm{m}} & 0 \\
0 & I_{n-\mathrm{m}}
\end{array}\right] .
$$

By reversing the order of the operands we get $P^{-1} \cdot P=B B^{+}+$ $B^{\perp} B^{\perp+}=I_{n}$.
Now, we can project the perturbation $\phi$ into the matched and unmatched spaces

$$
\phi=\phi_{\mathrm{m}}+\phi_{\mathrm{u}} \quad \phi_{\mathrm{m}} \triangleq B B^{+} \phi \quad \phi_{\mathrm{u}} \triangleq B^{\perp} B^{\perp+} \phi
$$

where $\phi_{\mathrm{m}}$ and $\phi_{\mathrm{u}}$ are the components that belong to the matched and unmatched spaces, respectively.

To determine the motion equations at the sliding manifold we use the equivalent control method [1]. The derivative of $s$ along time is

$$
\begin{aligned}
\dot{s} & =G\left[f+B\left(u_{0}+u_{1}\right)+B B^{+} \phi+B^{\perp} B^{\perp+} \phi\right]-G\left[f+B u_{0}\right] \\
& =G B\left(u_{1}+B^{+} \phi\right)+G \phi_{\mathrm{u}}
\end{aligned}
$$

The equivalent control is obtained by solving the equation $\dot{s}=0$ for $u_{1}$

$$
u_{1 \mathrm{eq}}=-B^{+} \phi-(G B)^{-1} G \phi_{\mathrm{u}}
$$

Remark 1: In the majority of the papers dealing with SMC, perturbations are assumed to be matched and the term on the far right is usually ignored.

By substituting $u_{1 \text { eq }}$ for $u_{1}$ in (1), we obtain the sliding dynamics

$$
\begin{aligned}
\dot{x}_{\mathrm{eq}} & =f+B\left(u_{0}-B^{+} \phi-(G B)^{-1} G \phi_{\mathrm{u}}\right)+B B^{+} \phi+B^{\perp} B^{\perp+} \phi \\
& =f+B u_{0}+\left[I-B(G B)^{-1} G\right] \phi_{\mathrm{u}} .
\end{aligned}
$$

From the last equation, we can draw several conclusions. First, the dynamics at the sliding manifold do not contain the matched perturbation: It has been successfully rejected. Second, with respect to conventional SMC, we have gained some extra degrees of freedom. We can use $u_{0}$ to stabilize the nominal system and to treat the unmatched perturbation. The projection matrix $G$ can now be considered a free parameter. Third, the order of the equivalent dynamics is equal to that of the original system, that is, there is no order reduction. This is the "price" we pay in return for the extra degrees of freedom and the elimination of the reaching phase. Fourth, the unmatched perturbation is now multiplied by a matrix

$$
\Gamma \triangleq\left[I-B(G B)^{-1} G\right]
$$

Another way to look at this, is that we have traded the original perturbation $\phi_{\mathrm{m}}+\phi_{\mathrm{u}}$, for a new one: $\phi_{\mathrm{eq}} \triangleq \Gamma \phi_{\mathrm{u}}$.

\section{Specific Questions}

Matrix $\Gamma$ is the main concern of this note. We would like to pose two specific questions regarding $\Gamma$.

1) Is there a $G^{*}$, such that norm of the equivalent perturbation $\phi_{\text {eq }}$ is minimal?

2) Does matrix $\Gamma$ amplify the unmatched perturbation? i.e., is the norm of $\phi_{\mathrm{eq}}$ greater than the norm of $\phi_{\mathrm{u}}$ ?

These questions make sense whenever we are considering unmatched perturbations and $u_{0}$ is to be designed with robustness against unmatched uncertainty in mind.

\section{MAIN RESULTS}

In this section, we answer the questions formulated in the problem statement and make some comments on the answers.

Proposition 2: $B^{T}$ is a matrix which minimizes the norm of $\phi_{\mathrm{eq}}$, i.e.,

$$
G^{*}=B^{T}=\arg \min _{G \in \mathbb{R}^{m \times n}}\left\|\left[I-B(G B)^{-1} G\right] \phi_{\mathrm{u}}\right\|_{2} .
$$


Proof: Notice first that

$$
\left\|\left[I-B(G B)^{-1} G\right] \phi_{\mathrm{u}}\right\|_{2}=\left\|\phi_{\mathrm{u}}-B \varphi\right\|_{2}
$$

where $\varphi=(G B)^{-1} G \phi_{\mathrm{u}}$. Thus, (6) can be rewritten in the form

$$
\varphi^{*}=\arg \min _{\varphi \in \mathbb{R}^{m}}\left\|\phi_{\mathrm{u}}-B \varphi\right\|_{2}
$$

which, according to the Projection theorem [16, p. 51] has $\varphi^{*}=B^{+} \phi_{\mathrm{u}}$ as a solution. Making $G=B^{T}$, we will have

$$
\varphi=\left(B^{T} B\right)^{-1} B^{T} \phi_{\mathrm{u}}=B^{+} \phi_{\mathrm{u}}=\varphi^{*}
$$

which implies (6).

Notice that for $G=B^{+}$we also have $\varphi=B^{+} \phi_{\mathrm{u}}$, so $B^{+}$also minimizes $\phi_{\mathrm{eq}}$.

Proposition 3: Given $m<n$ and a minimizing $G^{*}$, the resulting $\Gamma^{*}=I-B\left(B^{+} B\right)^{-1} B^{+}=I-B B^{+}$has euclidean norm equal to one. Moreover, the resulting equivalent perturbation $\phi_{\text {eq }}$ is equal to the original unmatched perturbation $\phi_{\mathrm{u}}$.

Proof: Notice first that

$$
\begin{aligned}
\Gamma^{* T} \Gamma^{*} & =\left[I-B B^{+}\right]\left[I-B B^{+}\right] \\
& =I-B B^{+}-B B^{+}+B B^{+} B B^{+} \\
& =I-B B^{+}=\Gamma^{*}
\end{aligned}
$$

which means that $\Gamma^{*}$ is a symmetric matrix and therefore all the eigenvalues are real. Suppose that $v$ is an eigen-vector associated to any eigen-value $\lambda$ of $\Gamma^{*}$, that is

$$
\Gamma^{*} v=\lambda v \quad \Rightarrow \quad v^{T} \Gamma^{* T} \Gamma^{*} v=\lambda^{2}\|v\|^{2} .
$$

However, since $\Gamma^{* T} \Gamma^{*}=\Gamma^{*}$, we have

$$
v^{T} \Gamma^{* T} \Gamma^{*} v=v^{T} \Gamma^{*} v=\lambda\|v\|^{2} .
$$

From (7) and (8), it is clear that the eigen-values of $\Gamma$ must satisfy $\lambda^{2}=\lambda$. The last equation has two solutions, $\lambda=0$ and $\lambda=1$. Since $\operatorname{rank}\left(B B^{+}\right)<n$, the rank of $I-B B^{+}$cannot be zero. This means that $\Gamma^{*}$ must have at least one eigen-value different from zero, that is, the maximum eigen-value is one. The last sentence implies that $\left\|\Gamma^{*}\right\|=1$.

The second statement of the proposition follows directly from the fact that at $G=B^{+}$(or $G=B^{T}$ ) the product $G \phi_{\mathrm{u}}$ equals zero.

A possible interpretation of the previous theorems is that in order to avoid amplification we should only penalize the difference between the actual and nominal trajectories, projected into the matched space. Notice that for $G=B^{+}$the equivalent control (4) becomes $u_{1 \mathrm{eq}}=$ $-B^{+} \phi$, so, it should only contain the matched perturbations: Any attempt to compensate the unmatched perturbations with the discontinuous control would only make matters worst.

The selection $G=B^{+}$has other advantages. First, the discontinuous control (3) is simplified to

$$
u_{1}=-\rho \frac{s}{\|s\|}
$$

Second, the gain $\rho$ and as a consequence the amplitude of the chattering can be reduced. Consider the candidate Lyapunov function $V=$ $\|s\|^{2} / 2$. At $G=B^{+}$the derivative of $s$ is

$$
\dot{s}=-\rho \frac{s}{\|s\|}+B^{+} \phi
$$

and the derivative of $V$ is

$$
\dot{V}=s^{T}\left(-\rho \frac{s}{\|s\|}+B^{+} \phi\right) \leq-\|s\|\left(\rho-\left\|B^{+} \phi\right\|\right) .
$$

In order to guarantee the sliding motion the discontinuous action only has to major the matched disturbance. In the general case, the derivative of the Lyapunov function is

$$
\begin{aligned}
\dot{V} & =s^{T}\left(G B\left(-\rho \frac{(G B)^{T} s}{\left\|(G B)^{T} s\right\|}+B^{+} \phi\right)+G \phi_{\mathrm{u}}\right) \\
& \leq-\left\|(G B)^{T} s\right\|\left(\rho-\left\|B^{+} \phi-(G B)^{-1} G \phi_{\mathrm{u}}\right\|\right) .
\end{aligned}
$$

So it is reasonable to select $G=B^{+}$. We close this section by writing the dynamics at the sliding manifold

$$
\dot{x}_{\mathrm{eq}}=f+B u_{0}+\phi_{\mathrm{u}} .
$$

\section{CASE OF STUdY: ISM AND $\mathcal{H}_{\infty}$ CONTROL}

In this section, we analyze the specific combination of ISMC and another robust method. The main goal of this section is to support the previous analysis and Propositions 2 and 3. For simplicity, we have chosen a linear technique: $\mathcal{H}_{\infty}$ control.

\section{A. Background, $\mathcal{H}_{\infty}$ Control}

Within the classical framework, when the full state is available the plants under consideration have the form

$$
\begin{aligned}
& \dot{x}=A x+B_{w} w+B u \\
& z=C x+D u, \quad z \in \Re^{n+m}
\end{aligned}
$$

where $z$ is an artificial penalty variable, matrices $C$ and $D$ are of appropriate dimension and establish a compromise between the cost associated to the state and the cost of the control used to keep the state within some bounds. The goal is to minimize the $\mathcal{H}_{\infty}$ norm of the transfer matrix $T_{z w}$ that goes from $w$ to $z$.

The following assumption is typical.

Assumption 4: $(A, B)$ is stabilizable, $(C, A)$ is detectable and $D^{T}\left[\begin{array}{ll}C & D\end{array}\right]=\left[\begin{array}{ll}0 & I\end{array}\right]$.

The first part of Assumption 4 is obvious and the second guarantees the boundedness of the state. The last part means that $z$ has no cross weighting between the state and control, and that the control weight matrix is the identity. The latter can be relaxed by a suitable coordinate transformation.

The following theorem (given without proof) is a standard result of $\mathcal{H}_{\infty}$ control [17].

Theorem 1 (Doyle et al.): Given Assumption 4, there exist a controller satisfying

$$
\left\|T_{z w}\right\|_{\infty}<\gamma
$$

iff there exists a real, symmetric, positive-semidefinite matrix $X$ satisfying the Riccati equation

$$
X A+A^{T} X-X\left(B B^{T}-\gamma^{-2} B_{w} B_{w}^{T}\right) X+C^{T} C=0 .
$$

Moreover, when this condition holds, one such controller is

$$
u=-B^{T} X x .
$$

In [18] and [19], it is shown that the $\mathcal{H}_{\infty}$ norm in the frequency domain and the (truncated) $\mathcal{L}_{2}$ induced norm of a linear system in the 
time domain are equivalent, i.e., if the conditions of Theorem 1 are satisfied, then

$$
\int_{t_{0}}^{T}\|z\|^{2} \mathrm{~d} \tau \leq \gamma^{2} \int_{t_{0}}^{T}\|w\|^{2} \mathrm{~d} \tau
$$

holds for all $T \geq t_{0}$. This equivalence allows to understand the $\mathcal{H}_{\infty}$ problem in terms of disturbance attenuation, to generalize the $\mathcal{H}_{\infty}$ control objective to nonlinear systems and to restate the $\mathcal{H}_{\infty}$ control problem in the following terms: Minimize the system's performance index, where the performance index $\gamma$, is understood as a truncated $\mathcal{L}_{2}$ gain.

\section{B. Proposed Methodology}

The basic idea is to use an ISMC to reject the matched perturbation and design the nominal control using $\mathcal{H}_{\infty}$ techniques to attenuate the unmatched one. Suppose that a control is to be designed for system (12). In terms of (1) we have $f(x, t)=A x$ and $\phi=B_{w} w$. According to (11), the system's dynamics at the sliding manifold is $\dot{x}=A x+B^{\perp} B^{\perp+} B_{w} w+B u_{0}$, where $\phi_{\mathrm{u}}=B^{\perp} B^{\perp+} B_{w} w$ was used to derive the previous equation. Notice that the discontinuous control $u_{1}$ is already fixed, so we need to replace $u$ by $u_{0}$ in the definition of the penalty variable $z$, that is

$$
z_{0}=C x+D u_{0} .
$$

The problem now becomes that of finding a minimum $\gamma$ and a semidefinite matrix $X$ that satisfies (13), but with $B_{w}$ substituted by $B^{\perp} B^{\perp+} B_{w}$.

The control $u_{1}$ is used to keep the state within some bounds and the cost of it should be taken into account if a comparison with the standard $\mathcal{H}_{\infty}$ control strategy is to be made, in other words: For comparison purposes the original definition of $z$ should be used. Whether or not the discontinuous control $u_{1}$ improves the over all performance index is not an easy question to answer, for it depends mainly on the weight $C$ assigned to the state. We can however, make a (rather informal) remark: notice that by orthogonality $\left\|B^{\perp} B^{\perp+} B_{w} w\right\|^{2}=\left\|B_{w} w\right\|^{2}-\left\|B B^{+} B_{w} w\right\|^{2}$. Since the squared norm of the unmatched perturbation is not bigger than the original one, we should expect a better performance index if the weight given to the state is "high-enough."

We summarize the proposed methodology in the following algorithm.

1) Solve the Riccati equation

$$
X A+A^{T} X-X\left(B B^{T}-\gamma^{-2} \bar{B}_{w} \bar{B}_{w}^{T}\right) X+C^{T} C=0
$$

where $\bar{B}_{w} \triangleq B^{\perp} B^{\perp+} B_{w}$.

2) Set the sliding manifold as

$$
s=B^{+}\left[x(t)-x\left(t_{0}\right)-\int_{t_{0}}^{t}\left(A-B B^{T} X\right) x(\tau) \mathrm{d} \tau\right] .
$$

3) Set the control as

$$
u=-B^{T} X x-\rho \frac{s}{\|s\|} \quad \rho>\left\|B^{+} B_{w} w\right\| .
$$

\section{Numerical Example}

Consider the following LTI system:

$$
\begin{aligned}
{\left[\begin{array}{l}
\dot{x}_{1} \\
\dot{x}_{2} \\
\dot{x}_{3} \\
\dot{x}_{4}
\end{array}\right]=\left[\begin{array}{cccc}
0 & 0 & 1 & 0 \\
0 & 0 & 0 & 1 \\
-1 & 0 & -2 & 0 \\
2 & -1 & 0 & 2
\end{array}\right] } & {\left[\begin{array}{l}
x_{1} \\
x_{2} \\
x_{3} \\
x_{4}
\end{array}\right] } \\
& +\underbrace{\left[\begin{array}{ll}
0 & 0 \\
0 & 0 \\
0 & 1 \\
1 & 0
\end{array}\right]}_{B_{w}}\left[\begin{array}{l}
w_{1} \\
w_{2}
\end{array}\right]+\underbrace{\left[\begin{array}{l}
0 \\
0 \\
0 \\
1
\end{array}\right]}_{B} u .
\end{aligned}
$$

We define the error variable as

$$
z_{0}=\underbrace{\left[\begin{array}{c}
\operatorname{diag}(5,5,10,10) \\
\mathbf{0}
\end{array}\right]}_{C} x+\underbrace{\left[\begin{array}{l}
\mathbf{0} \\
1
\end{array}\right]}_{D} u_{0} .
$$

1) $\mathcal{H}_{\infty}$ Control Alone: Equation (13) has

$$
X=\left[\begin{array}{cccc}
70.35 & -2.43 & 20.53 & 2.24 \\
-2.43 & 57.62 & -7.17 & 4.29 \\
20.53 & -7.17 & 70.46 & -0.47 \\
2.24 & 4.29 & -0.47 & 12.83
\end{array}\right]
$$

as a solution, where the optimal value $\gamma=5.9337$ was calculated up to four decimal places. The resulting controller is then

$$
u=-B^{T} X x=\left[\begin{array}{llll}
-2.24 & -4.29 & 0.47 & -12.82
\end{array}\right] x .
$$

2) ISMC Plus $\mathcal{H}_{\infty}$ : The disturbances are first decomposed as

$$
B_{w} w=\underbrace{\left[\begin{array}{ll}
0 & 0 \\
0 & 0 \\
0 & 0 \\
1 & 0
\end{array}\right]}_{B B+B_{w}} w+\underbrace{\left[\begin{array}{ll}
0 & 0 \\
0 & 0 \\
0 & 1 \\
0 & 0
\end{array}\right]}_{B^{\perp} B^{\perp+B} w} w .
$$

The first part is matched and will be eliminated by the discontinuous control $u_{1}$; the second is unmatched and will be treated using the continuous control $u_{0}$, designed using the $\mathcal{H}_{\infty}$ technique.

The solution to (16) is given by

$$
X=\left[\begin{array}{cccc}
70.25 & -2.34 & 20.41 & 2.17 \\
-2.34 & 57.39 & -6.94 & 4.23 \\
20.41 & -6.94 & 70.34 & -0.45 \\
2.17 & 4.23 & -0.45 & 12.61
\end{array}\right] \text { and } \gamma=5.9291
$$

The nominal control is

$$
u_{0}=-B^{T} X x=\left[\begin{array}{llll}
-2.17 & -4.23 & 0.45 & -12.61
\end{array}\right] x
$$

and the sliding manifold is

$$
s(x, t)=B^{+}\left[x(t)-x\left(t_{0}\right)-\int_{t_{0}}^{t}\left(A x+B u_{0}\right) \mathrm{d} \tau\right] .
$$

\section{Simulation Results}

Three simulations were carried out. In all cases the system was perturbed by the signal

$$
w=\cos (\pi t)\left[\begin{array}{ll}
1 & -0.6
\end{array}\right]^{T}
$$



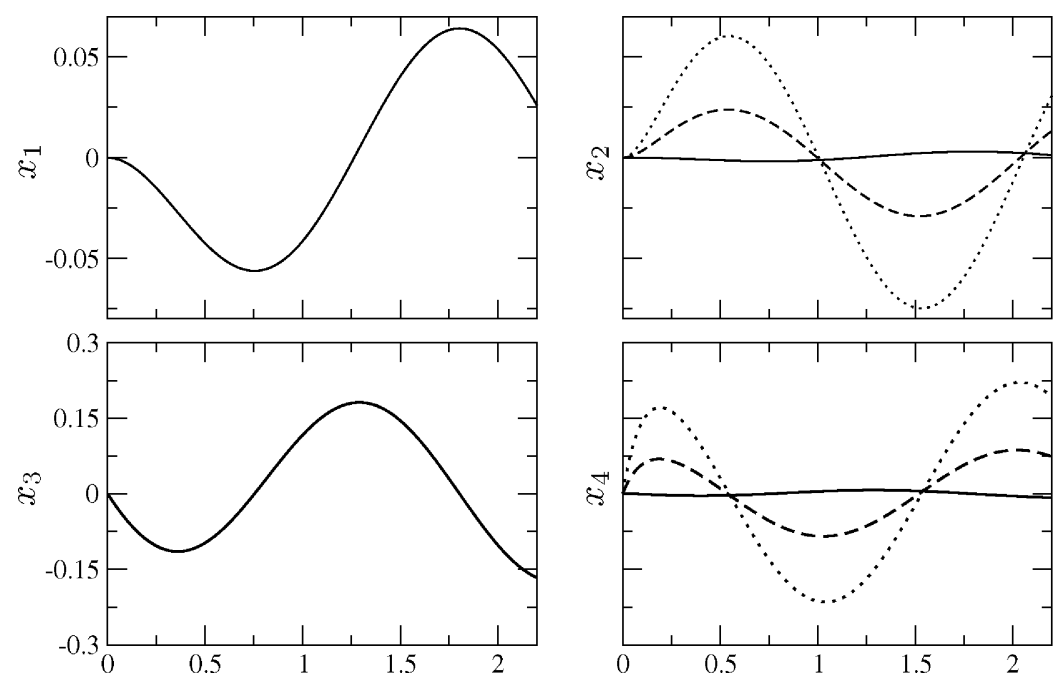

Fig. 1. System's states. Simulation results for the $\mathcal{H}_{\infty}$ controller (dashed-line) and the "ISM plus $\mathcal{H}_{\infty}$ " controller for two cases: $G \neq B+($ dotted-line) and $G=B^{+}$(solid-line). The time histories of $x_{1}$ and $x_{3}$ are too similar to appreciate a difference.

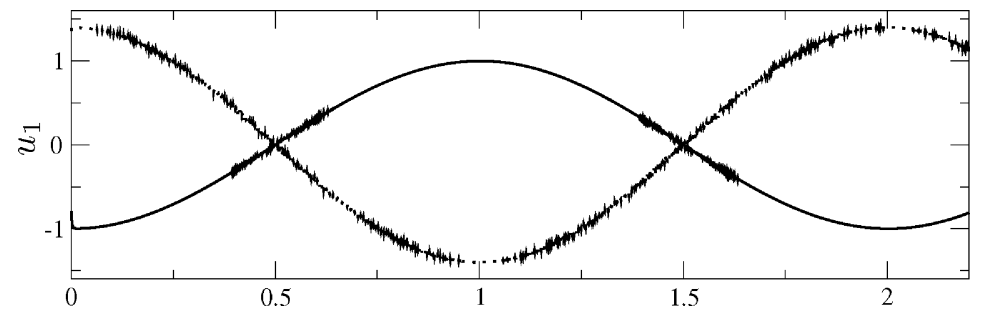

Fig. 2. High frequency controls. $u_{1}=-3.6 s /(|s|+0.0001)$ for $G \neq B^{+}$(dotted) and $u_{1}=-1.2 s /(|s|+0.0001)$ for $G=B^{+}$(solid).

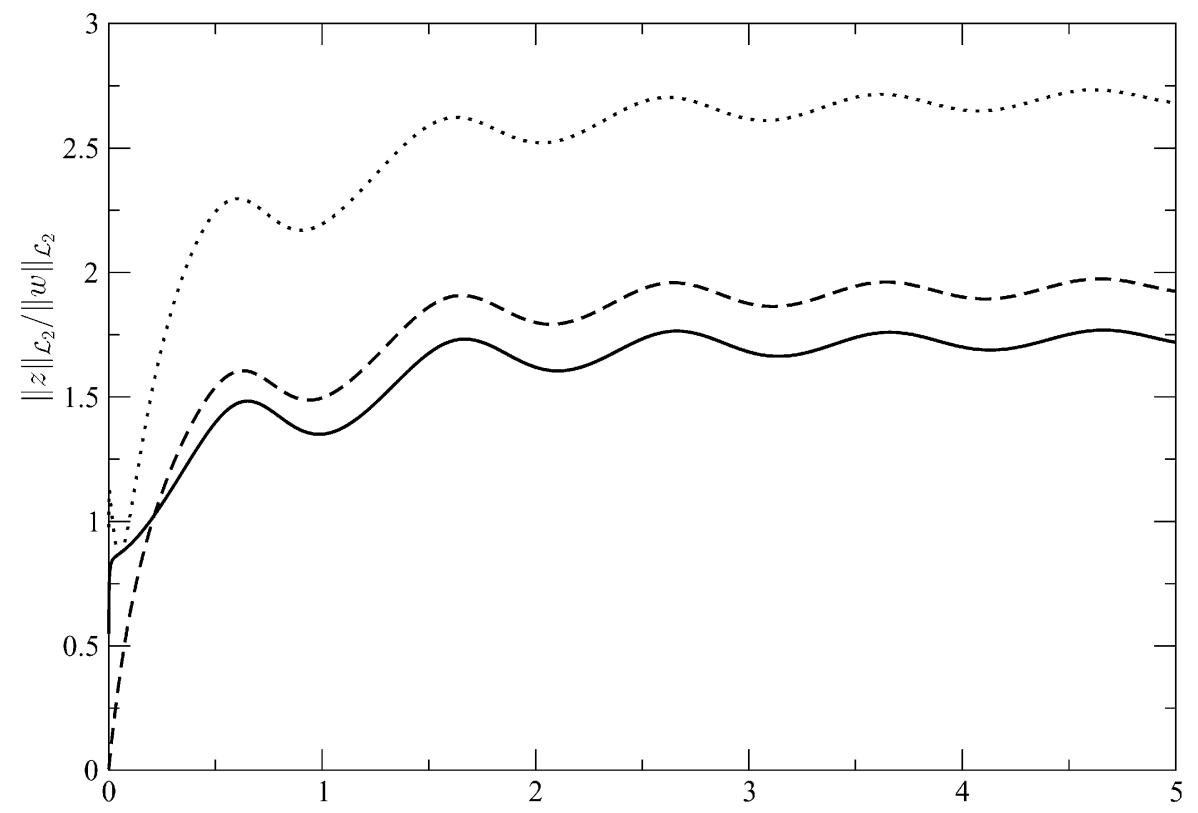

Fig. 3. Actual values of the $\mathcal{L}_{2}$ gains for perturbations (18).

and the initial conditions were set at the origin. The first simulation was made using the $\mathcal{H}_{\infty}$ controller. The second one was made using the combination "ISMC plus $\mathcal{H}_{\infty}$," but with $G$ set different from $B^{+}$

$$
G=\left[\begin{array}{llll}
2 & 2 & 2 & 0.5
\end{array}\right]
$$

The third simulation was made using the optimal value $G=B^{+}$. The system's states are shown in Fig. 1. The time histories of $x_{1}$ and $x_{3}$ are the same in all cases because the control has no influence on them.
Notice, however, that for $G=B^{+}$there is an attenuation of the amplitudes of $x_{2}$ and $x_{4}$ with respect to the $\mathcal{H}_{\infty}$ controller and there is an increase of the amplitudes when $G \neq B^{+}$.

In the second simulation the gain $\rho$ needed to enforce the sliding mode was obtained using (10) and was set to 3.6. In the last simulation it was obtained using (9) and was set to 1.2. The discontinuous controls were approximated by

$$
u_{1}=-3.6 \frac{s}{|s|+0.0001} \quad \text { and } \quad u_{1}=-1.2 \frac{s}{|s|+0.0001}
$$


respectively. It can be seen in Fig. 2 that when $G \neq B^{+}$the control acts in the opposite direction, i.e., it's effect is counter effective.

For comparison purposes, we have in Fig. 3 a plot of $\|z\|_{\mathcal{L}_{2}} /\|w\|_{\mathcal{L}_{2}}$ for each controller. When $G$ is selected improperly, the value is increased due to the amplification of $\phi_{\mathrm{u}}$. When $G$ is selected properly, the value is, after a short transient, lower than the one obtained by $\mathcal{H}_{\infty}$ alone, even though the discontinuous component was included in the penalty variable (i.e., $z=C x+D\left(u_{0}+u_{1}\right)$ ).

\section{CONCLUSION}

In this note, we studied the effects that the projection matrix has on the resulting (equivalent) perturbation. It was shown that in the presence of unmatched disturbances the projection matrix of an ISM controller should be selected carefully, for the resulting controller could amplify them. Two propositions provide a way for selecting the projection matrix correctly. The proposed parameters ensure that the effect of the unmatched disturbance will not be amplified by the discontinuous control. It is also shown that the discontinuous control can not attenuate the unmatched disturbances.

The analysis is aimed at combining ISMC with other robust techniques. $\mathcal{H}_{\infty}$ control was selected as a specific case, but other techniques could be used as well. Simulation results support the analysis developed.

\section{REFERENCES}

[1] V. Utkin, J. Guldner, and J. Shi, Sliding Modes in Electromechanical Systems. London, U.K.: Taylor and Francis, 1999.

[2] C. Edwards and S. K. Spurgeon, "Sliding mode stabilization of uncertain systems using only output information," Int. J. Control, vol. 62, pp. 1129-1144, 1995.

[3] K. Bag, S. K. Spurgeon, and C. Edwards, "Output feedback sliding mode design for linear uncertain systems," Proc. Inst. Elect. Eng. Control Theory Appl., vol. 144, pp. 209-216, May 1997.

[4] C. Edwards, A. Akoachere, and S. K. Spurgeon, "Sliding-mode output feedback controller design using linear matrix inequalities," IEEE Trans. Autom. Control, vol. 46, no. 1, pp. 115-119, Jan. 2001.

[5] A. Levant, "Higher-order sliding modes, differentiation and output-feedback control," Int. J. Control, vol. 76, pp. 924-941, 2003.

[6] Y. B. Stessel, "Nonlinear output tracking in conventional and dynamic sliding manifolds," IEEE Trans. Autom. Control, vol. 42, no. 9, pp. 1282-1286, Sep. 1997.

[7] A. Poznyak, Y. B. Stessel, and C. Jiménez, "Mini-max sliding mode control for multimodel linear time varing systems," IEEE Trans. Autom. Control, vol. 48, no. 12, pp. 2141-2150, Dec. 2003.

[8] Y. Orlov, W. Perruquetti, and J. Richard, "Sliding mode control synthesis of uncertain time-delay systems," Asian J. Control, vol. 5, pp. 568-577, 2003.

[9] H. H. Choi, "An LMI-based switching surface design method for a class of mismatched uncertain systems," IEEE Trans. Autom. Control, vol. 48, no. 9, pp. 1634-1638, Sep. 2003.

[10] G. P. Matthews and R. A. DeCarlo, "Decentralized tracking for a class of interconnected nonlinear systems using variable structure control," Automatica, vol. 24, pp. 187-193, 1988.

[11] V. Utkin and J. Shi, "Integral sliding mode in systems operating under uncertainty conditions," in Proc. Conf. Decision and Control, Kobe, Japan, Dec. 1996, pp. 4591-4596.

[12] A. Poznyak, L. Fridman, and F. J. Bejarano, "Mini-max integral sliding mode control for multimodel linear uncertain systems," IEEE Trans. Autom. Control, vol. 49, no. 1, pp. 97-102, Jan. 2004.

[13] L. Fridman, A. Poznyak, and F. J. Bejarano, "Mini-max multimodel optimal problem via integral sliding mode control," in Proc. Amer. Control Conference, Boston, MA, Jun. 2004, pp. 620-625.

[14] J. Xu, Y. Pan, and T. Lee, "Analysis and design of integral sliding mode control based on Lyapunov's direct method," in Proc. Amer. Control Conf., Denver, CO, Jun. 2003, pp. 192-196.
[15] M. Basin, J. Rodríguez-González, L. Fridman, and P. Acosta, "Integral sliding mode design for robust filtering and control of linear stochastic time-delay systems," Int. J. Robust Nonlinear Control, vol. 15, pp. 407-421, 2005.

[16] D. G. Luenberger, Optimization by Vector Space Methods. New York: Wiley, 1969.

[17] J. C. Doyle, P. P. Khargonekar, and B. A. Francis, "State-space solutions to $\mathcal{H}_{2}$ and $\mathcal{H}_{\infty}$ control problems," IEEE Trans. Autom. Control, vol. 34 no. 8, pp. 831-847, Aug. 1989.

[18] A. J. van der Schaft, " $\mathcal{L}_{2}$-gain analysis of nonlinear systems and nonlinear state feedback $\mathcal{H}_{\infty}$ control," IEEE Trans. Autom. Control, vol. 37, no. 6, pp. 770-784, Jun. 1992.

[19] A. Isidori and A. Astolfi, "Disturbance attenuation and $\mathcal{H}_{\infty}$-control via measurement feedback in nonlinear systems," IEEE Trans. Autom. Control, vol. 37, no. 9, pp. 1283-1293, Sep. 1992.

\section{Adaptive Finite-Time Control of Nonlinear Systems With Parametric Uncertainty}

\author{
Yiguang Hong, Jiankui Wang, and Daizhan Cheng
}

Abstract-In this note, global finite time stabilization is investigated for a class of nonlinear systems in $\boldsymbol{p}$ normal form with parametric uncertainties. To achieve finite-time stabilization, a constructive control design approach is proposed by following backstepping methodology, and an adaptive finite-time control law is obtained in the form of continuous time-invariant feedback.

Index Terms-Adaptive control, finite-time stability, nonsmooth feedback, settling time.

\section{INTRODUCTION}

Nonsmooth finite-time control can make the controlled systems to reach their targets in a finite time. It was first studied in the literature of optimal control. In recent years, finite-time stabilization controllers have been investigated for a number of class of systems [2], [4], [5], [7]-[9]. In particular, [5] has constructed finite-time stabilizing laws for the nonlinear systems in $p$ normal form without parametric uncertainties. Indeed, asymptotic/exponential stabilizations are sufficient for many engineering applications. For very demanding applications, finite-time stabilization offers an effective alternative, which yields, in some sense, fast response, high tracking precision, and disturbance-rejection properties because of their nonsmoothness [2], [8]. The studies of finite-time control can show us how to increase the precision in a given settling time or make the system convergent fast to the target within arbitrary given precision.

Uncertainties do exist in any real world systems. Adaptive control is one of the effective ways to deal with control systems with parametric uncertainty. Although it is not easy to propose adaptive control strategies for general nonlinear systems, a great deal of efforts have been made in this area and some well-known adaptive design methods are proposed for nonlinear systems with uncertain parameters (referring

Manuscript received August 24, 2004; revised April 11, 2005, October 26, 2005, and January 13, 2006. Recommended by Associate Editor S. S. Ge. This work was supported by the NNSF of China under Grants 60425307, 50595411, and 10472129 .

The authors are with the Key Laboratory of Systems and Control, Institute of Systems Science, Chinese Academy of Sciences, Beijing 100080, China.

Digital Object Identifier 10.1109/TAC.2006.875006 